\title{
Delivery Timing and Cesarean Delivery Risk in Women with Mild Gestational Diabetes
}

\begin{abstract}
Amelia L. SUTTON, M.D., Ph.D., Lisa MELE, Sc.M., Mark B. LANDON, M.D., Susan M. RAMIN, M.D., Michael W. VARNER, M.D., John M. THORP Jr., M.D., Anthony SCISCIONE, D.O., Patrick CATALANO, M.D., Margaret HARPER, M.D., M.Sc., George SAADE, M.D., Steve N. CARITIS, M.D., Yoram SOROKIN, M.D., and William A. GROBMAN, M.D., M.B.A. for the Eunice Kennedy Shriver National Institute of Child Health and Human Development (NICHD) Maternal-Fetal Medicine Units (MFMU) Network

Departments of Obstetrics and Gynecology of the University of Alabama at Birmingham, Birmingham, AL; The George Washington University Biostatistics Center, Washington, DC; The Ohio State University, Columbus, $\mathrm{OH}$; The University of Texas Health Science Center at Houston, Houston, TX; University of Utah, Salt Lake City, UT; University of North Carolina at Chapel Hill, Chapel Hill, NC; Drexel University, Philadelphia, PA; Case Western Reserve University-MetroHealth Medical Center, Cleveland, $\mathrm{OH}$; Wake Forest University Health Sciences, Winston-Salem, NC; University of Texas Medical Branch, Galveston, TX; University of Pittsburgh, Pittsburgh, PA; Wayne State University, Detroit, MI; and Northwestern University, Chicago, IL
\end{abstract}

\begin{abstract}
OBJECTIVE-To evaluate the relationship between gestational age (GA) and induction of labor (IOL) and the rate of cesarean delivery (CD) in women with mild gestational diabetes (GDM).

STUDY DESIGN—Secondary analysis of data from a multi-center RCT of mild GDM treatment. $\mathrm{CD}$ rate of women delivering at term ( 237 weeks) was evaluated using two complementary approaches: 1) IOL $v s$. spontaneous labor: women induced at each GA compared with those who spontaneously labored at the same GA, and 2) IOL $v s$. expectant management: women delivered after IOL at each GA compared with those delivering after spontaneous labor at the same GA or subsequently after spontaneous or induced labor (outcome at each week compared with expectant management at that week). Logistic regression adjusted for potential confounders.
\end{abstract}

RESULTS-The overall CD rate was 13\%. When compared to 39 weeks (either IOL or spontaneous labor) as the referent, there was no significant difference in the CD rate in women delivered at 37, 38, or 40 weeks. However, IOL was associated with a 3-fold increase in CD rate

(C) 2014 Mosby, Inc. All rights reserved.

Correspondence: Amelia L.M. Sutton M.D., Ph.D., 176F 10270C, $61919^{\text {th }}$ St S, Birmingham, AL 35249-7333, Phone: 205-975-2423, Fax 205-975-9858 alsutton@uabmc.edu.

Disclosure: The authors report no conflict of interest.

Presented in part at the $32^{\text {st }}$ Annual Meeting of the Society for Maternal-Fetal Medicine in Dallas, Texas February 6-11, 2012.

Publisher's Disclaimer: This is a PDF file of an unedited manuscript that has been accepted for publication. As a service to our customers we are providing this early version of the manuscript. The manuscript will undergo copyediting, typesetting, and review of the resulting proof before it is published in its final citable form. Please note that during the production process errors may be discovered which could affect the content, and all legal disclaimers that apply to the journal pertain. 
at 41 weeks and beyond as compared with IOL at 39 weeks. Similarly, there was a 3-fold increase in $\mathrm{CD}$ rate in women who were induced when compared to those managed expectantly at 40 completed weeks.

CONCLUSIONS-Induction of labor in women with mild gestational diabetes mellitus (GDM) does not increase the rate of cesarean delivery prior to 40 weeks gestation.

\section{Keywords}

cesarean delivery; gestational diabetes; induction of labor

\section{INTRODUCTION}

The cesarean delivery rate has continued to rise to a recent high of $32 \%$, increasing more than $50 \%$ in the last decade ${ }^{1}$. Similarly, the rates of IOL have increased, now affecting $23 \%$ of all births ${ }^{1}$. Previous observational studies have suggested that induction predisposes women to $\mathrm{CD}$. Specifically, when comparing women undergoing IOL to those experiencing spontaneous labor, an increased rate of $\mathrm{CD}$ has been observed. ${ }^{2,3}$ However, when compared to expectant management, elective induction of labor at or beyond 41 weeks is associated with a decreased rate of cesarean section. ${ }^{4}{ }^{5}$ A recent Cochrane metanalysis suggests that elective induction of labor at term, when compared with expectant management, is associated with a decreased rate of cesarean section as well as other adverse perinatal outcomes. ${ }^{6}$

The discrepancies in the findings of these are partially explained by differences in comparison groups. While initial studies compared induced with spontaneous labor, more recent studies have used an expectant management comparison group, which includes patients who spontaneously delivered as well as those who were induced at later gestational ages. This comparison is more appropriate in clinical decision-making since expectant management, not spontaneous labor, at a particular gestational age is the only alternative.

The MFMU Network randomized controlled trial (RCT) for the treatment of mild GDM demonstrated that treatment of mild gestational diabetes (GDM) is associated with a lower rate of cesarean delivery $(26.9 \%$ vs. $33.8 \%, p=0.02) .{ }^{7}$ This reduced risk was apparent even after excluding cesarean indications such as malpresentation, placenta previa, oligohydramnios, and previous cesarean. A high proportion (27\%) of patients underwent induction in each group.

The primary aim of this study was to compare the rate of CD by gestational age and by IOL vs. expectant management in women with GDM. As a secondary aim, we compared perinatal outcomes among the study groups.

\section{METHODS AND MATERIALS}

We performed a secondary analysis of a multicenter randomized trial evaluating the effect of treatment in women with mild GDM. ${ }^{7}$ In the original trial, women at 24-30 weeks gestation with blood glucose levels of $135-199 \mathrm{mg} / \mathrm{dL}$ following a 50 gram glucose loading test were invited to enroll. Eligible women underwent a blinded 3-hour 100 gram oral glucose 
tolerance test. Mild GDM was defined as fasting glucose levels of less than $95 \mathrm{mg} / \mathrm{dL}$ and at least two abnormal timed measurements $(>180 \mathrm{mg} / \mathrm{dL}$ at 1 hour, $>155 \mathrm{mg} / \mathrm{dL}$ at 2 hours, and $>140 \mathrm{mg} / \mathrm{dL}$ at 3 hours). Women with mild GDM were randomized to usual prenatal care or treatment with dietary modifications, glucose monitoring, and insulin, if indicated. Providers and patients were blinded to GDM diagnosis in the standard care group. The original study was approved by the institutional review boards of all participating clinical centers, and all enrolled women gave informed consent.

Women in both the treatment and standard care group were included in this secondary analysis if they delivered at term ( 37 weeks), underwent induced or spontaneous labor, and had cephalic presentations. Patients were excluded from analysis if they had an elective scheduled $\mathrm{CD}$, had one or more previous CDs, had a non-cephalic presentation, or a major fetal anomaly. The primary outcome was $\mathrm{CD}$. The $\mathrm{CD}$ rate was assessed by two approaches: 1) patients delivered at a specific GA (in completed weeks) and type of labor were compared to those who delivered at 39 weeks; 2) patients undergoing IOL at a specific GA were compared with those "expectantly managed", which included patients who delivered at the same GA following spontaneous labor and patients who delivered at subsequent GA following spontaneous or induced labor. We examined several secondary outcomes. First, the composite perinatal outcome, included any one of the following: hypoglycemia, hyperbilirubinemia, RDS, and birth trauma which have been associated with GDM. We also assessed the frequency of admission of the infant to the neonatal intensive care unit (NICU), of birthweight $>4000 \mathrm{~g}$, and of large-for-gestational age (LGA) infants, defined as birthweight $>90^{\text {th }}$ percentile. $^{8}$

Categorical variables were analyzed using the chi square or Fisher's exact test. Continuous variables were analyzed using the Wilcoxon rank sum or the Kruskal-Wallis test. Study outcomes by completed GA week were calculated by type of labor for the two comparison methods. Multivariable logistic regression analysis was used to adjust for potential confounders for most outcomes including maternal age, race/ethnicity, pre-pregnancy body mass index (BMI), parity, smoking status, Cesarean delivery (for neonatal outcomes), infant sex (for neonatal outcomes), timing of dating ultrasound (trimester), SGA, and treatment group. No adjustments were made for the outcome of NICU admissions due to to small numbers. Adjusted odds ratios (OR) and 95\% confidence intervals (CI) were estimated relative to the IOL group at 39 weeks in method 1 and the expectant management group at each GA in method 2.

Statistical analyses were conducted using SAS software (SAS Institute, Cary, NC). A nominal two-sided $\mathrm{p}$-value $<0.05$ was considered for statistical significance with no adjustments made for multiple comparisons.

\section{RESULTS}

Of the 958 women enrolled in the original RCT, 679 met inclusion and exclusion criteria for this secondary analysis. Baseline characteristics of the study population are shown in Table I. Reflective of the original study population, over half of the women were Hispanic, and the 
mean BMI at enrollment was approximately $30( \pm 5.0) \mathrm{kg} / \mathrm{m}^{2}$. Race/ethnicity and GDM screen results differed by completed GA at delivery.

The overall rate of $\mathrm{CD}$ was $13 \%$. The indications for cesarean section were: failed induction (69.3\%), non-reassuring fetal tracing $(26.1 \%)$, cord prolapse (3.4\%), and chorioamnionitis (1.2\%). The crude and adjusted results for CD are presented in Table II. Of note, the results were adjusted for maternal age, ethnicity, BMI, parity, smoking, timing of dating ultrasound, SGA status, and assigned control $v s$. treatment group. The CD incidence increased from $10.3 \%$ to $22.7 \%$ as gestational age at delivery increased from 37 to 41 completed weeks. This pattern was primarily restricted to the IOL group. When comparing the rate of CD at each GA with 39 completed weeks as the referent (method 1), there was no significant difference in the adjusted CD delivery rate at 37,38 , and 40 completed weeks. However, at 41 weeks, IOL was associated with a 3 -fold increase in CD as compared with IOL at 39 weeks. Likewise, when comparing women undergoing IOL $v s$. those expectantly managed (method 2), IOL was not associated with an increased adjusted rate of CD at 37, 38, or 39 completed weeks. At 40 completed weeks, IOL was associated with a 3-fold increased in $\mathrm{CD}$ rate. There was a similar increase in $\mathrm{CD}$ rate at 41 completed weeks and greater, but this association was not statistically significant. The $\mathrm{CD}$ risks when stratified by treatment group are shown in Tables IIIA and IIIB. In the treated group, based on small numbers, there was a trend toward a reduction in CD risk associated with IOL prior to 39 weeks the OR (95\% CI) with IOL when compared to those expectantly managed was $1.97(0.39,9.95)$ at 40 and 3.94 $(0.10,158.6)$ at $\geq 41$ weeks (Table IIIB). However, these comparisons were not statistically significant. In the standard care group, the OR (95\% CI) for IOL compared to the expectant management group was $3.07(1.05,8.96)$ at 40 weeks and $3.76(0.69,20.58)$ at $\geq 41$ weeks; there were no increases at 37,38 or 39 weeks (Table IIIA).

The frequency and adjusted odds of the composite perinatal outcome were not different in the IOL group when compared to either the spontaneous labor (method 1) or expectant management group (method 2) at all GAs (Table IV). There were no cases of stillbirth or neonatal deaths in this study. Furthermore, there were no significant differences in the unadjusted frequency of NICU admissions between the groups with the exception of an increase in NICU admissions in the IOL group at $\geq 41$ weeks (there were no NICU admissions in the $\geq 41$ week expectant management group; data not shown). There was a significant increase in the frequency of infants with birthweight $>4000 \mathrm{~g}$ at $\geq 41$ weeks when compared to 39 weeks (OR of 2.83 with 95\% CI of 1.31-6.14) in all deliveries (data not shown). However, there were no significant differences in the frequency of either infants with birthweight $>4000 \mathrm{~g}$ or LGA neonates in the IOL vs. expectant management groups at all gestational ages (data not shown).

\section{COMMENT}

We found an increasing trend in cesarean delivery risk with increasing gestational age at term. When compared to expectant management, IOL in patients with mild GDM was not associated with an increase in CD rate until 40 weeks. When stratified for treatment group (standard care and treated groups), results (limited by numbers), were similar to the overall findings; the increase in CD risk from 40 weeks appeared to be more prominent among 
those who were not treated. Furthermore, we found no significant differences in perinatal outcomes between patients undergoing IOL compared to either those spontaneously laboring or those expectantly managed. No significant differences were also observed for NICU admissions, birthweight $>4000$, or LGA neonates.

A recent study indicates that IOL at 39 in women with GDM confers a decrease in perinatal mortality. ${ }^{9}$ Our results are mostly consistent with a previous cohort study of term deliveries that showed no difference in CD rate when IOL was compared to expectant management at all gestational ages. Similarly, a randomized controlled trial by Boulvain and colleagues showed no increase in $\mathrm{CD}$ rate in non-diabetic women undergoing IOL with suspected fetal macrosomia. ${ }^{10}$

Our findings are in line with metanalyses that showed no increase in the CD rate in women undergoing elective IOL at term. ${ }^{5,6}$ In our study, there were no differences in CD rates in women undergoing IOL $v s$. expectant management prior to 40 weeks. However, IOL was associated with an increase in CD rate from 40 weeks. As the population in the current study was restricted to women with mild GDM and included women undergoing both elective and indicated IOL, our findings could potentially be attributed to the higher risk of macrosomia, labor dystocia, and fetal heart rate abnormalities in gestational diabetics leading to higher $\mathrm{CD}$ rate with advancing gestation.

Previous studies have differed in the composition of the expectant management group. For example, Caughey et al. defined the expectant management group as women delivering, after either spontaneous or induced labor, during the week following the induction group. ${ }^{11}$ This approach may favor the induction group because it excludes women who spontaneously labor in the same week from the expectant management group. In contrast, Glantz included women who delivered following spontaneous labor the same week as the induction group in the expectant management group. ${ }^{12}$ In contrast to the current study, that analysis demonstrated a small but significant increased risk of CD associated with labor induction. ${ }^{12}$ We chose to define the expectant management group as women who delivered at the same GA following spontaneous labor and patients who delivered at subsequent GA following spontaneous or induced labor. These differences may account for some of the variations in findings among these studies. Additionally, given the limitations of the precision of dating, some of these differences may be affected by variability in the assignment of gestational age.

Although we simulate IOL $v s$. expectant management, prospective management regarding timing of delivery was not a focus of the primary trial. Therefore, this secondary analysis does not fully capture prospective management intent. Additionally, while adjustment for confounders such as parity did not alter the results, we do not have data on other factors, such as cervical status, that may affect these findings. We also did not take into account the type of gestational diabetes given that few women required medication. Our population is made up of only women with mild GDM, limiting the generalizability of findings to all patients with GDM. 
The overall $\mathrm{CD}$ rate in this selected population was relatively low at $13 \%$. The $\mathrm{CD}$ rate in original trial was $30 \%$, and the population selected for the current study excluded women with scheduled CDs, prior CDs, and non-cephalic presentations. It is not clear how a more liberal use of $\mathrm{CD}$ in this population would affect our findings. Over half of the patients in this study were Hispanic, reflective of the increased risk of GDM associated with Hispanic ethnicity. Finally, as this was a retrospective analysis of an existing trial, our sample size likely did not provide sufficient power to account for all possible differences in comparison groups.

As gestation progresses, particularly in pregnancies complicated by diabetes or other comorbidities, the risks of adverse perinatal outcomes such as stillbirth, fetal heart rate abnormalities, macrosomia, and labor dystocia increases. ${ }^{13}, 14$ The current study was limited in that there were no stillbirths in this group, which is explained by the relatively small sample size of women with only mild GDM.

Taken together, these data support the hypothesis that a policy of induction prior to 40 weeks in women with GDM may not increase the rate of cesarean delivery. Considered collectively with the studies associating decreasing adverse pregnancy outcomes with increasing gestational age, our findings suggest that delivery prior to 40 weeks, perhaps as early as 38 weeks, may improve CD risk in women with mild GDM. This should be weighed against the impact on other important perinatal outcomes. Therefore, a large, randomized controlled trial is warranted to further evaluate the maternal and neonatal risks and benefits of IOL at term and, thus, to determine the optimal gestational age of delivery in women with GDM.

\section{Acknowledgments}

The project described was supported by grants from the Eunice Kennedy Shriver National Institute of Child Health and Human Development (NICHD) [HD27915, HD34116, HD40485, HD34208, HD27869, HD40500, HD40560, HD34136, HD40544, HD27860, HD40545, HD53097, HD21410, HD27917, HD40512, HD53118, HD36801], General Clinical Research Centers Grant [M01-RR00034] and the National Center for Research Resources [UL1RR024989, M01-RR00080, UL1-RR025764, C06-RR11234] and does not necessarily represent the official views of the NICHD or NIH.

The authors would like to thank Alan T. N. Tita, M.D., Ph.D. for guidance in study design, data analysis, and manuscript preparation; Dr. John C. Hauth for assistance in study design; Francee Johnson, R.N., B.S.N. and JoAnn Tillinghast, R.N., M.S.N. for protocol development and coordination between clinical research centers; Elizabeth Thom, Ph.D., for protocol development/data management and statistical analysis; and Marshall W. Carpenter, M.D., and Catherine Y. Spong, M.D. for protocol development and oversight.

\section{References}

1. MARTIN JA, HAMILTON BE, VENTURA SJ, et al. Births: final data for 2009. National vital statistics reports : from the Centers for Disease Control and Prevention, National Center for Health Statistics, National Vital Statistics System. 2011; 60:1-70.

2. VAHRATIAN A, ZHANG J, TROENDLE JF, SCISCIONE AC, HOFFMAN MK. Labor progression and risk of cesarean delivery in electively induced nulliparas. Obstetrics and gynecology. 2005; 105:698-704. [PubMed: 15802393]

3. LUTHY DA, MALMGREN JA, ZINGHEIM RW. Cesarean delivery after elective induction in nulliparous women: the physician effect. American journal of obstetrics and gynecology. 2004; 191:1511-5. [PubMed: 15547518] 
4. HANNAH ME, HANNAH WJ, HELLMANN J, HEWSON S, MILNER R, WILLAN A. Induction of labor as compared with serial antenatal monitoring in post-term pregnancy. A randomized controlled trial. The Canadian Multicenter Post-term Pregnancy Trial Group. The New England journal of medicine. 1992; 326:1587-92. [PubMed: 1584259]

5. CAUGHEY AB, SUNDARAM V, KAIMAL AJ, et al. Systematic review: elective induction of labor versus expectant management of pregnancy. Annals of internal medicine. 2009; 151:252-63. W53-63. [PubMed: 19687492]

6. GULMEZOGLU AM, CROWTHER CA, MIDDLETON P, HEATLEY E. Induction of labour for improving birth outcomes for women at or beyond term. The Cochrane database of systematic reviews. 2012; 6:CD004945. [PubMed: 22696345]

7. LANDON MB, SPONG CY, THOM E, et al. A multicenter, randomized trial of treatment for mild gestational diabetes. The New England journal of medicine. 2009; 361:1339-48. [PubMed: 19797280]

8. ALEXANDER GR, KOGAN MD, HIMES JH. 1994-1996 U.S. singleton birth weight percentiles for gestational age by race, Hispanic origin, and gender. Maternal and child health journal. 1999; 3:225-31. [PubMed: 10791363]

9. ROSENSTEIN MG, CHENG YW, SNOWDEN JM, NICHOLSON JM, DOSS AE, CAUGHEY $\mathrm{AB}$. The risk of stillbirth and infant death stratified by gestational age in women with gestational diabetes. American journal of obstetrics and gynecology. 2012; 206:309, e1-7. [PubMed: 22464068]

10. BOULVAIN MSM-V, ROZENBERG P, IRION O. Induction of labor or expectant management for large-for-dates fetuses: a randomized controlled trial. American journal of obstetrics and gynecology. 2012; 206:S2.

11. CAUGHEY AB, NICHOLSON JM, CHENG YW, LYELL DJ, WASHINGTON AE. Induction of labor and cesarean delivery by gestational age. American journal of obstetrics and gynecology. 2006; 195:700-5. [PubMed: 16949399]

12. GLANTZ JC. Term labor induction compared with expectant management. Obstetrics and gynecology. 2010; 115:70-6. [PubMed: 20027037]

13. SMITH GC. Life-table analysis of the risk of perinatal death at term and post term in singleton pregnancies. American journal of obstetrics and gynecology. 2001; 184:489-96. [PubMed: 11228508]

14. RAND L, ROBINSON JN, ECONOMY KE, NORWITZ ER. Post-term induction of labor revisited. Obstetrics and gynecology. 2000; 96:779-83. [PubMed: 11042317]

\section{Appendix}

In addition to the authors, other members of the Eunice Kennedy Shriver National Institute of Child Health and Human Development Maternal-Fetal Medicine Units Network are as follows:

University of Texas Southwestern Medical Center - K. Leveno, L. Moseley, J. Gold, D. Bradford, L. Fay, M. Garcia, F. Capellan

Columbia University — R. Wapner, M. Miodovnik, F. Malone, S. Bousleiman, H. Husami, V. Carmona, N. Fredericks, E. Gantioqui, B. Greenspan, M. Williams

University of Utah — K. Anderson (University of Utah Health Sciences Center), P. Ashby (University of Utah Health Sciences Center), S. McAllister (University of Utah Health Sciences Center), S. Quinn (LDS Hospital), A. Guzman (McKay-Dee Hospital), F. Castinella (LDS Hospital), J. Steiner (McKay-Dee Hospital), J. Parker (Utah Valley Regional Medical Center) 
University of Alabama at Birmingham — J. Sheppard, J. Tisdale, A. Northen, D. Rouse, W. Andrews

Brown University — M. Carpenter, D. Catlow, D. Allard, M. Seebeck, J. Tillinghast

The Ohio State University — J. Iams, F. Johnson, C. Latimer, E. Weinandy, B. Maselli

University of North Carolina at Chapel Hill - K. Dorman, S. Brody, S. Timlin, J. Bernhardt

Drexel University - M. Hoffman, E. Guzman, M. Talucci, T. Grossman, C. Perez, L.

Zeghibe, P. Tabangin

Case Western Reserve University-MetroHealth Medical Center - B. Mercer, B. Stetzer, C. Milluzzi, W. Dalton, S. Pichette

Wake Forest University Health Sciences — M. Swain, P. Meis, J. White

The University of Texas Health Science Center at Houston - L. Gilstrap, K. Cannon, J. Martinez, D. Dusek

University of Texas Medical Branch - J. Moss, J. Brandon, A. Jackson, G. Hankins, D. Sharp

University of Pittsburgh - M. Bickus, H. Birkland, M. Cotroneo, N. Cuddy

Wayne State University — G. Norman, P. Lockhart, S. Blackwell, L. Quast

Northwestern University — P. Simon, G. Mallett

Oregon Health \& Science University — J. Tolosa, L. Davis, E. Lairson, C. Cromett, C. Naze, M. Blaser

The George Washington University Biostatistics Center - E. Thom, J. Zachary, B. Getachew, C. Cobb, L. Leuchtenburg, S. Gilbert

Eunice Kennedy Shriver National Institute of Child Health and Human Development - C. Spong, S. Tolivaisa, K. Howell

The MFMU Network Steering Committee Chair (University of Texas Medical Branch, Galveston, TX) - G.D. Anderson, M.D. 


\section{CLINICAL IMPLICATIONS}

- Induction of labor, compared to expectant management, prior to 40 weeks does not increase cesarean delivery risk in women with mild gestational diabetes mellitus (GDM).

- Large randomized controlled trials are warranted to fully evaluate the maternal and neonatal risks associated with induction of labor and to determine the optimal gestational age of delivery in women with mild GDM. 


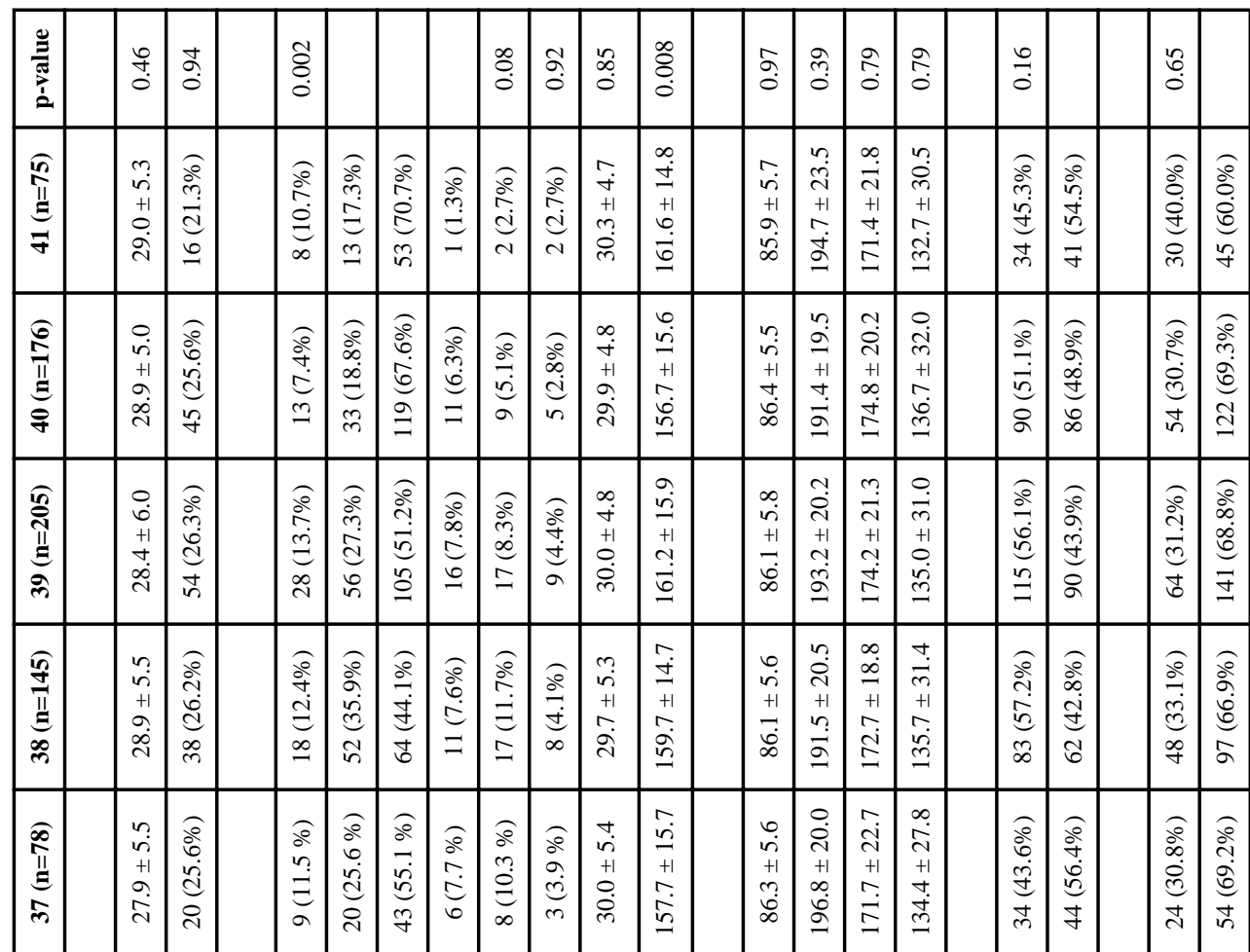

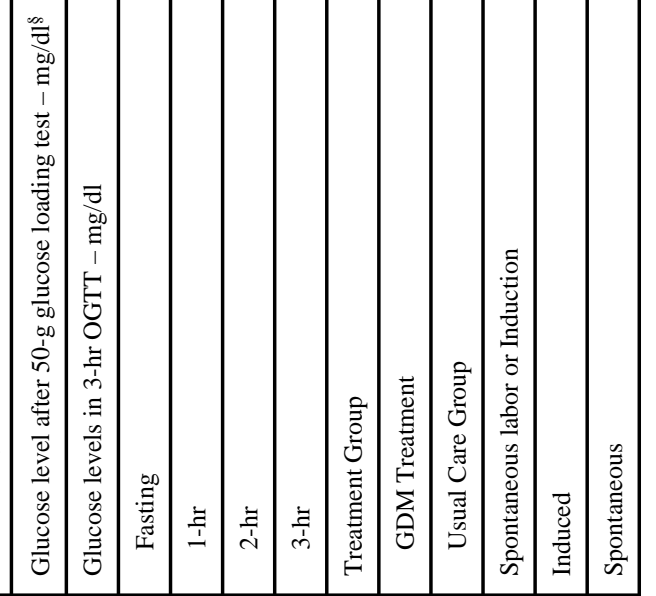




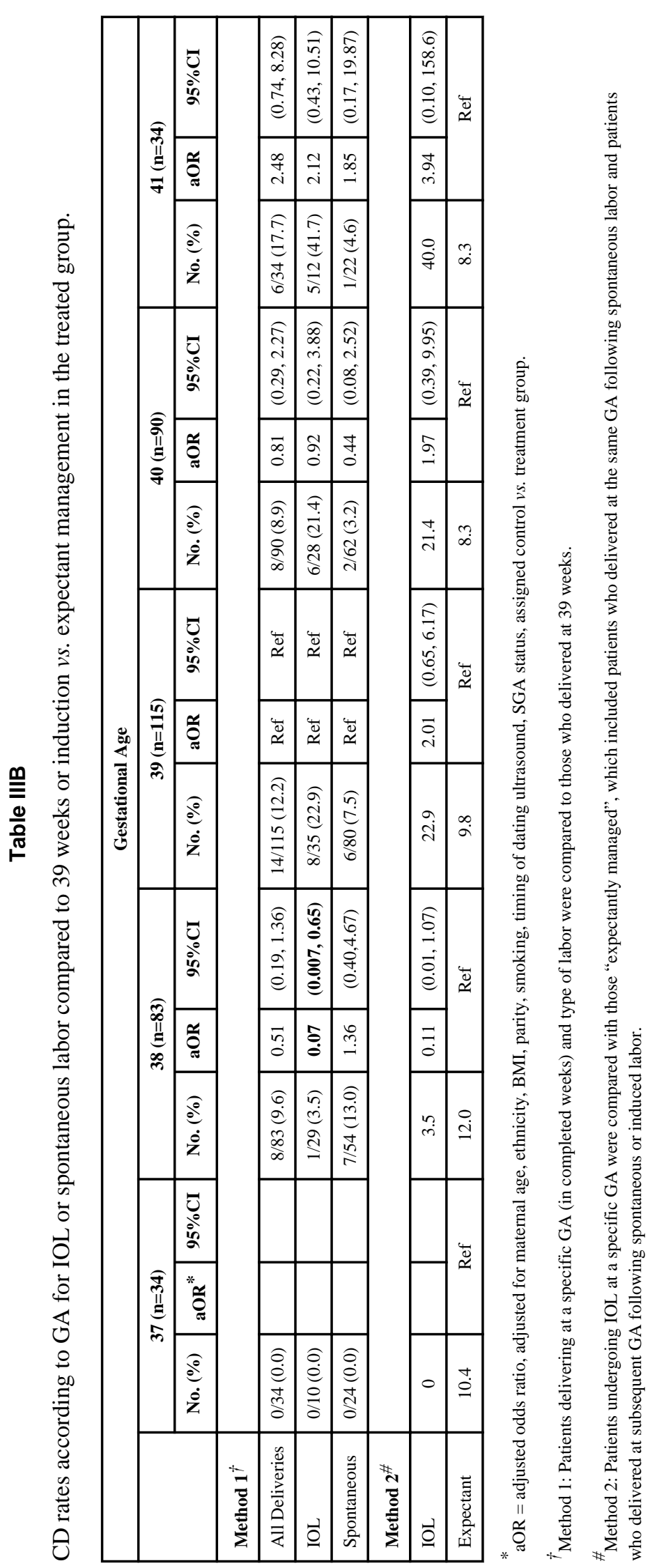

Am J Obstet Gynecol. Author manuscript; available in PMC 2015 September 01. 


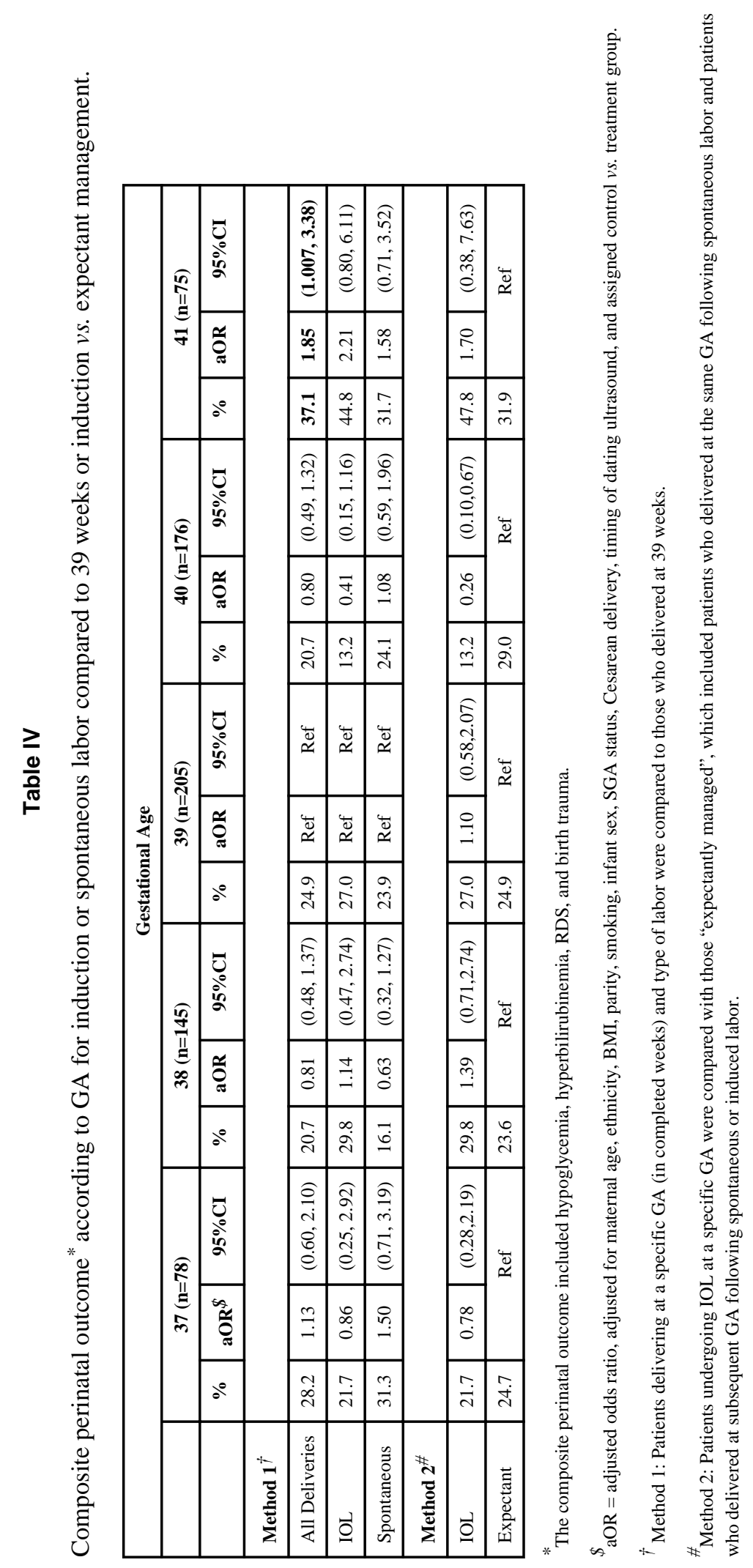

